\title{
Hyperprolactinemia and body weight: prevalence of obesity and overweight in patients with hyperprolactinemia
}

\author{
Julia Fernanda Semmelmman Pereira-Lima, Carolina Garcia Soares Leães, Flávio Maciel Freitas Neto, Malu Viter da Rosa Barbosa, \\ Ana Luiza Mattos da Silva and Miriam da Costa Oliveira* \\ *Correspondence: mco@portoweb.com.br \\ Center for Neuroendocrinology, Universidade Federal de Ciências da Saúde de Porto Alegre (UFCSPA), Rua Sarmento Leite, 245, \\ 90050-170, Porto Alegre, RS, Brazil.
}

\begin{abstract}
Background and objectives: Recent studies have shown an association between prolactin (Prl) levels and body weight, with increased prevalence of obesity in patients with prolactinomas and weight loss associated with hyperprolactinemia. However, data on variations in body weight associated with different degrees of hyperprolactinemia remain scarce. This study aimed to evaluate the prevalence of obesity and overweight in patients with hyperprolactinemia of various causes and correlate Prl levels with body mass index (BMI).

Methods: Medical records of patients with hyperprolactinemia were retrospectively reviewed. Patients were classified according to BMI as normal weight (19.0-24.9), overweight (25-29.9), and class I (30-34.9), class II (35-39.9) and class III (> 40) obesity. Disease etiology was divided into microprolactinoma, macroprolactinoma, and other causes for analysis.

Results: Of 138 eligible patients, 52.9\% had micro or macroprolactinoma and $65.2 \%$ were overweight or obese. Mean patient age was $36.2 \pm 13.6$ years. Obesity was more prevalent in patients with macroprolactinoma (50\%), and overweight in patients with other causes of hyperprolactinemia (37.9\%), with no statistical difference between groups. Prl levels were higher in macroprolactinomas, with a statistically significant difference $(\mathrm{p}<0.001)$ compared to microprolactinomas and other causes of hyperprolactinemia. Prl was also significantly higher in microprolactinomas than in other causes of hyperprolactinemia $(\mathrm{p}<0.001)$.

Conclusions: Prevalence of obesity was significantly high in hyperprolactinemic patients, regardless of the degree of obesity and the cause of hyperprolactinemia. It is important to monitor BMI in patients with elevated Prl levels to introduce measures aiming to maintain an ideal weight and reduce associated comorbidities.
\end{abstract}

Keywords: Body weight, hyperprolactinemia, obesity, overweight, prolactinoma

\section{Introduction}

Prolactin (Prl) is a single-chain polypeptide involved in several actions, such as lactation, luteal function, reproduction, appetite, suppression of fertility, homeostasis, osmotic balance, immunity, and coagulation $[\mathbf{1 , 2}]$.

Prl receptor ( $\mathrm{Prl}-\mathrm{R})$ gene expression has already been described in adipose tissue, and an increase in this expression during lactation has been documented in rats and humans $[3,4]$. Prl-R-deficient mice have shown reduced abdominal fat and leptin concentration compared to controls [5]. In hamsters, inhibition of Prl secretion by bromocriptine has led to a reduction in fat deposition, without reducing food intake or body weight [2]. In human adipose tissue, Prl suppresses lipid storage and adipokine release and also has a paracrine/autocrine function in relation to adiponectin by binding to its receptors [6-8]. Prl increases the expression of adiponectin receptors, a hormone that is secreted by adipose tissue, increasing insulin sensitivity [9]. In humans, outside pregnancy, Prl secretion is altered by increasing body weight in adults and children. However, no molecular basis has been found which links Prl with increasing body fat, weight and appetite, although some data suggest the involvement of Prl with leptin [10].

In recent years, some studies have shown an increased prevalence of obesity in patients with prolactinomas [11,12], while others have reported weight loss [12-15] or weight gain after treatment of hyperprolactinemia $[16,17]$. However, there are no studies investigating variations in body weight associated with different degrees of hyperprolactinemia or their relation to the cause of hormone hypersecretion.

This study aimed to evaluate the prevalence of obesity and overweight in patients with hyperprolactinemia, whether or not associated with prolactinomas, and to correlate Prl levels with body mass index (BMI) in hyperprolactinemia of various causes.

\section{Materials and methods}

The medical records of consecutive treatment-naive patients with hyperprolactinemia attending the Center for Neuroendocrinology at Universidade Federal de Ciências da Saúde de Porto Alegre (UFCSPA), Brazil, between 2000 and 2010 were retrospectively reviewed. The study was approved by the local Ethics Committee (Institutional Review Board-equivalent) and was conducted in accordance with the provisions of the 
Table 1. Age, body mass index (BMI) and prolactin levels, according to the etiology of hyperprolactinemia (n=138).

\begin{tabular}{lccc}
\hline & Macroprolactinoma $(\mathbf{n}=\mathbf{4 6})$ & Microprolactinoma $(\mathbf{n}=\mathbf{2 7})$ & Other causes $(\mathbf{n}=\mathbf{6 5})$ \\
\hline Age $($ years $)$ & $36.6( \pm 13.6)$ & $34.6( \pm 12.7)$ & $37.4( \pm 14.6)$ \\
BMI $\left(\mathrm{kg} / \mathrm{m}^{2}\right)$ & $30.3( \pm 7.6)$ & $27.6( \pm 5.6)$ & $27.8( \pm 4.8)$ \\
Baseline prolactin $(\mathrm{ng} / \mathrm{mL})$ & $200(111-379)^{\mathrm{a}}$ & $75(39-149)^{\mathrm{b}}$ & $56(38-104)^{\mathrm{c}}$ \\
Maximum prolactin $(\mathrm{ng} / \mathrm{mL})$ & $348(198-1127)^{\mathrm{a}}$ & $192(108-260)^{\mathrm{b}}$ & $76(46-123)^{\mathrm{c}}$ \\
\hline
\end{tabular}

Age and BMI are expressed as mean \pm standard deviation (SD), and prolactin as median and interquartile range.

Different letters indicate statistical difference: $a \neq b(p<0.001) ; a \neq c(p<0.001) ; b \neq c(p<0.001)$.

Declaration of Helsinki.

Based on medical record review, 115 patients with prolactinoma and 82 patients with idiopathic or drug-induced hyperprolactinemia, both with negative imaging findings, had sufficient data for inclusion in the analysis.

Patients with prolactinoma were defined as those with significant hyperprolactinemia and pituitary lesions less than $1 \mathrm{~cm}$ (microprolactinomas) or greater than or equal to $1 \mathrm{~cm}$ (macroprolactinomas) who responded to dopamine agonists (normalization of Prl levels and/or radiographic evidence of significant decrease in lesion size). At the moment of the evaluation, no patient was under dopamine agonist therapy. Hyperprolactinemia was considered as serum Prl greater than the reference value for the method according to sex. Patients with a history of use of drugs that cause hyperprolactinemia and negative imaging findings, were considered as having hyperprolactinemia secondary to drugs, the patients without history of use of drugs causing hyperprolactinemia and negative imaging findings, were considered as patients with idiopathic hyperprolactinemia. Patients were classified according to BMI, as follows: normal weight (BMI 19.0-24.9); overweight (BMI 25-29.9); class I obesity (BMI 30-34.9); class II obesity (BMI 35-39.9); and class III obesity (BMI > 40).

Exclusion criteria were: primary hypothyroidism, polycystic ovary syndrome, chronic renal failure, cirrhosis, adrenal insufficiency, irritative lesions of the chest wall or spinal cord, pregnancy, breastfeeding, nipple manipulation, and physical or emotional stress. Patients receiving medical treatment for overweight or obesity, patients using dopamine agonists, and patients undergoing surgery or radiotherapy for treatment of prolactinoma were also excluded from analysis.

Data on sex, age, weight $(\mathrm{kg})$, height $(\mathrm{m}), \mathrm{BMI}\left(\mathrm{kg} / \mathrm{m}^{2}\right)$, serum Prl at diagnosis (baseline Prl) and maximum Prl level achieved during the patient's clinical course (maximum Prl) were evaluated.

Disease etiology was divided into microprolactinoma, macroprolactinoma, and other causes of hyperprolactinemia for analysis.

\section{Statistical analysis}

Quantitative data were expressed as mean and standard deviation, and in the presence of asymmetry, as median and interquartile range. Correlations were evaluated using analysis of variance (ANOVA) and the Spearman rank correlation. The
ANOVA was used followed by the Tukey test for multiple comparison. The level of significance was set at $5 \%$. Data were analyzed using the Statistical Package for the Social Sciences (SPSS), version 17.0.

\section{Results}

Of 197 medical records initially reviewed, 138 were eligible and analyzed for this report. Of these, 101 (73.2\%) were women and $37(26.8 \%)$ were men, and mean age was $36.2 \pm 13.6$ years. Regarding the etiology of hyperprolactinemia, 73 (52.9\%) patients had prolactinoma - 46 (63\%) with macroprolactinoma (31 women, $15 \mathrm{men}$ ) and 27 (37\%) with microprolactinoma (24 women, 3 men) - and 65 (47.1\%) had other causes of hyperprolactinemia (46 women, 19 men). Data on age, BMI, and Prl levels according to the etiology of hyperprolactinemia are described in Table 1. No differences were found in the data analyzed between women and men, and therefore they were analyzed together as a group. The patients diagnosed as having hyperprolactinemia secondary to drugs, the overwhelming majority (82\%) was related to neuroleptics and antidepressants.

Regarding body weight, $65.2 \%$ of patients were overweight or obese. The number of overweight and/or obese patients, according to the etiology of hyperprolactinemia, is shown in Table 2.

As for the etiology of hyperprolactinemia, there was a positive correlation between baseline and maximum Prl levels and pituitary tumor volume, with macroprolactinomas showing baseline and maximum Prl levels significantly higher than those of microprolactinomas and other causes of hyperprolactinemia $(p<0.001)$.

In relation to lipid and glycemic profile, no differences were found between the different groups according to the etiology of hyperprolactinemia and the correlations with prolactin levels. There was a significant but weak correlation of BMI with baseline Prl and maximum $\mathrm{Prl}\left(\mathrm{r}_{\mathrm{s}}=0.3\right)$, demonstrating that baseline $\operatorname{Prl}\left(p=0.014, r_{s}=0.21\right)$ and maximum $\operatorname{Prl}(p=0.013$, $r_{s}=0.21$ ) values increased as BMI increased. When analyzed separately according to the cause of hyperprolactinemia, only the microprolactinoma group showed a significant but moderate correlation between $\mathrm{BMI}$ and baseline $\operatorname{Prl}(\mathrm{p}=0.02$, $r_{\mathrm{s}}=0.45$ ). See Graph 1 and 2.

A statistically significant difference was also observed between groups classified according to $\mathrm{BMI}$, as follows: baseline Prl - normal weight and class III obese $(p=0.047)$, 
Pereira-Lima et al. Research Journal of Endocrinology and Metabolism 2013, http://www.hoajonline.com/journals/pdf/2053-3640-1-2.pdf

Table 2. Number of normal weight, overweight and obese patients, according to the etiology of hyperprolactinemia.

\begin{tabular}{lcccc}
\hline & Total $(\mathbf{n}=\mathbf{1 3 8})$ & Macroprolactinoma $(\mathbf{n}=\mathbf{4 6})$ & Microprolactinoma $(\mathbf{n}=\mathbf{2 7})$ & Other causes $(\mathbf{n}=\mathbf{6 5})$ \\
\hline Normal weight & $48(34.8 \%)$ & $15(30 \%)$ & $11(40 \%)$ & $19(30 \%)$ \\
Overweight & $42(30.4 \%)$ & $9(20 \%)$ & $8(30 \%)$ & $25(37.9 \%)$ \\
Obesity - class I & $24(17.4 \%)$ & $9(20 \%)$ & $5(20 \%)$ & $13(20 \%)$ \\
Obesity - class II & $16(11.6 \%)$ & $7(16 \%)$ & $2(6.7 \%)$ & $7(10.6 \%)$ \\
Obesity - class III & $8(5.8 \%)$ & $6(14 \%)$ & $1(3.3 \%)$ & $1(1.5 \%)$ \\
Overweight + obesity & $65.2 \%$ & $70 \%$ & $60 \%$ & $70 \%$ \\
\hline
\end{tabular}

BMI = body mass index.

Normal weight: BMI 19.0-24.9; overweight: BMI 25-29.9; obesity - class I: BMI 30-34.9; obesity - class II:

BMI 35-39.9; obesity - class III: BMI > 40
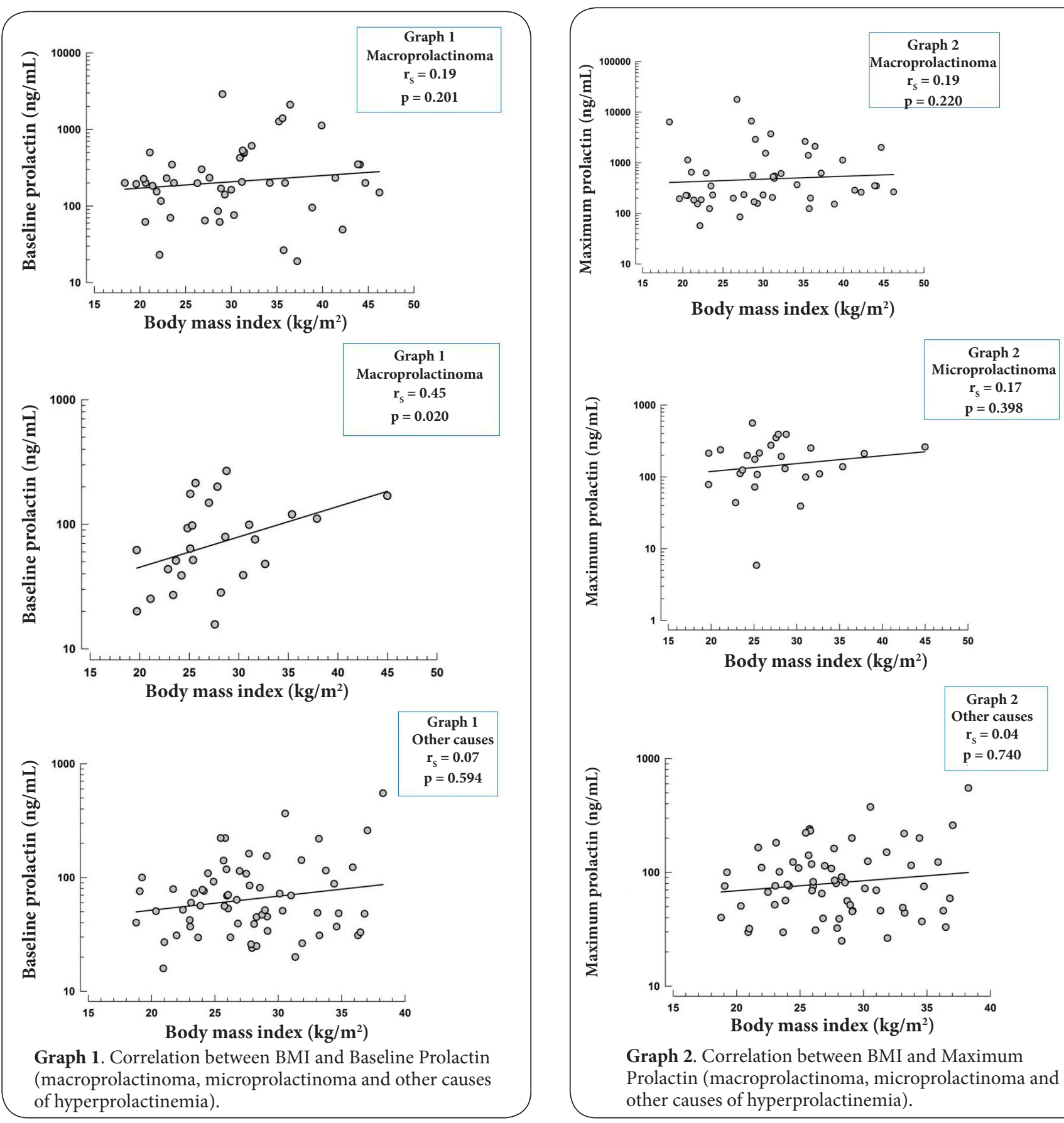

Graph 2. Correlation between BMI and Maximum

Prolactin (macroprolactinoma, microprolactinoma and other causes of hyperprolactinemia). 


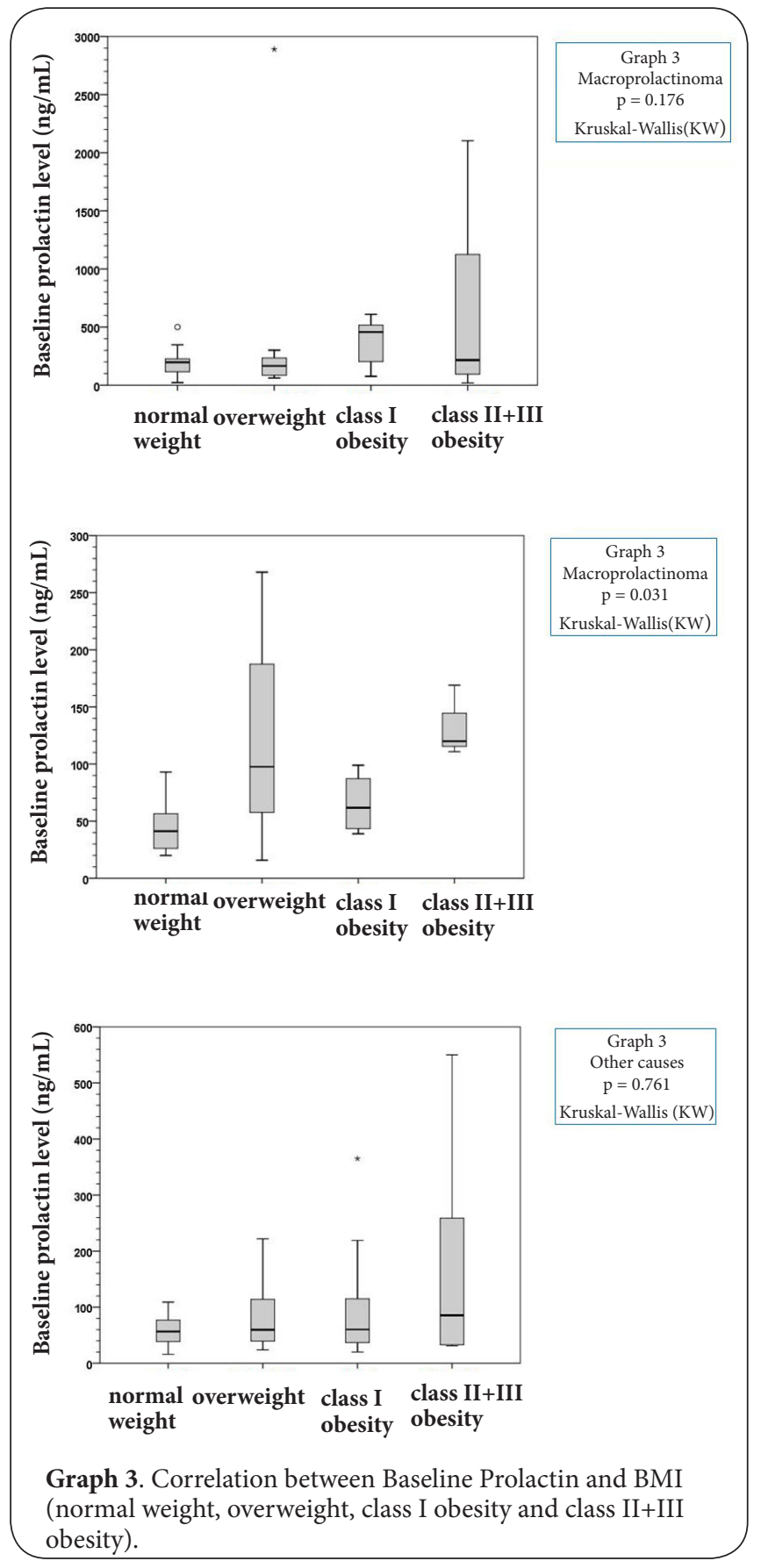

normal weight and class II+III obese $(p=0.03)$; maximum Prl - normal weight and overweight $(\mathrm{p}=0.02)$, normal weight and class III obese $(p=0.03)$, normal weight and class II+III obese $(p=0.02)$, overweight and class II+III obese $(p=0.02)$. In all comparisons, baseline Prl and maximum Prl values increased as BMI increased. See Graph $\mathbf{3}$ and 4.

\section{Discussion}

Hyperprolactinemia is one of the most common hypothalamicpituitary endocrine disorders among women of reproductive

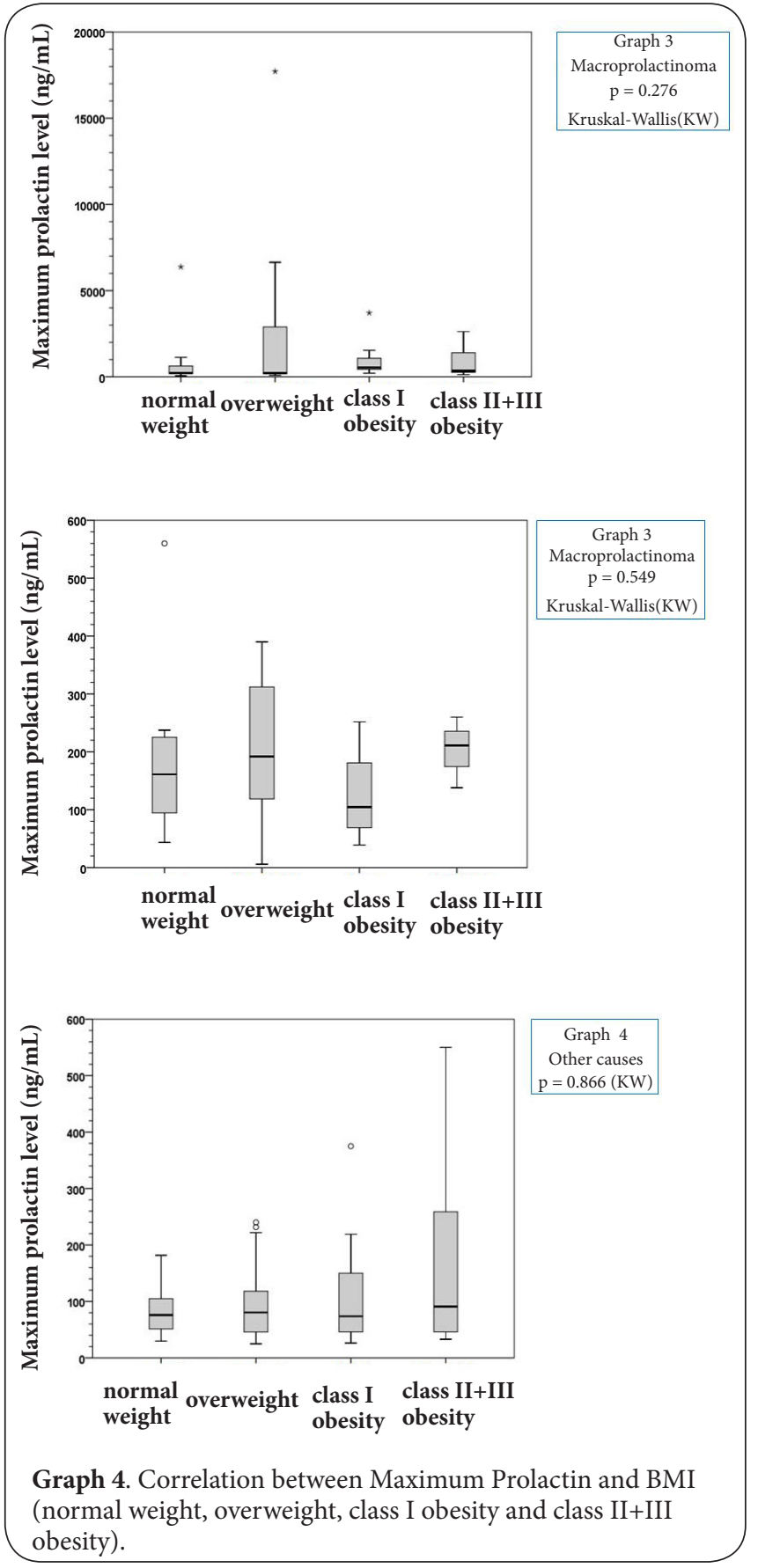

age. It often occurs associated with prolactinomas and secondary to the use of centrally acting drugs. In the absence of a known cause, hyperprolactinemia is considered idiopathic [2]. Women were more prevalent in our sample (73.2\%), with mean age of 36.27 years. More than half of the patients had prolactinoma as a cause of hyperprolactinemia. In patients with prolactinoma, serum Prl levels are generally proportional to the size of the prolactinoma, with macroprolactinomas exhibiting levels above $250 \mathrm{ng} / \mathrm{mL}$ [18]. Regarding druginduced hyperprolactinemia, Prl values vary greatly and are 
likely to reach levels similar to or higher than those reported for macroprolactinomas. Idiopathic hyperprolactinemia usually shows a moderate increase in Prl levels [19]. The overall etiologic distribution and associated Prl levels found in our study are very similar to data already reported in the literature $[17,18]$.

The number of obese people in Brazil continues to increase every year, becoming a serious public health problem. Data from the Brazilian Institute of Geography and Statistics (IBGE) show that, in $2006,42.7 \%$ of the population was overweight, increasing to $48.1 \%$ in 2010. In Brazil, obesity affected $11.4 \%$ of adults in 2006 and $15 \%$ in 2010 [20]. In the present study, $34.8 \%$ of hyperprolactinemic patients were obese, a $132 \%$ higher rate than that described for the general population of our country [20].

In a study describing the association of Prl and weight in 47 patients with prolactinoma, before treatment, over $50 \%$ of patients reported weight gain after the disease $(11.8 \pm 2.0 \mathrm{~kg})$, with mean weight of $83 \pm 2.4 \mathrm{~kg}$ and BMl of $27.3 \pm 0.6 \mathrm{~kg} / \mathrm{m}^{2}$ [11]. In another study, in which 42 patients with prolactinoma were evaluated and 36 individuals with clinically non-functioning adenomas (NFA) were used as controls, a high prevalence of obesity was also described in prolactinomas [12]. Recent weight gain ( 8 to $22 \mathrm{~kg}$ ) was found in $13 / 42$ patients with prolactinoma against only one in the control group. Mean weight was $93 \pm 3.4 \mathrm{~kg}$ in patients with prolactinoma and $78 \pm 2.7$ $\mathrm{kg}$ in NFA patients. In that study, weight gain and elevated body weight were frequently associated with prolactinomas [12]. In a study involving 22 patients with prolactinoma, with median Prl of $144 \mathrm{ng} / \mathrm{mL}$ and BMI of $29.5 \mathrm{~kg} / \mathrm{m}^{2}$, prevalence of obesity and overweight was $45 \%$ and $27 \%$, respectively [14]. In a study evaluating 37 patients with prolactinoma, patients with microprolactinoma had significantly lower initial body weight than those with macroprolactinoma $(p=0.006)$ [17].

In this study, $50 \%$ of patients with macroadenomas were obese and $20 \%$ were overweight. In patients with microadenomas, $30 \%$ were obese and $30 \%$ were overweight, and in cases of other causes of hyperprolactinemia, $32.1 \%$ of patients were obese and $37.9 \%$ were overweight. There was no difference in the incidence or duration of hypogonadism among the groups (microprolactinoma, macroprolactinoma, and other cases of hyperprolactinemia). There was a prevalence of obesity in patients with macroprolactinoma, compared to microprolactinoma and other causes of hyperprolactinemia. In a study of patients with antipsychotic-induced hyperprolactinemia, Prl levels were not correlated with BMI [21]. Likewise, in patients with prolactinoma, no correlation was found between Prl levels and BMI [14]. In a study comparing patients with prolactinoma and NFA, weight gain and elevated body weight were often associated with prolactinomas [12]. In the present study, a significant but weak correlation was found between Prl and BMI in all causes of hyperprolactinemia evaluated.

When examining the possible effect of obesity on the pathogenesis of hyperprolactinemia, which should be considered in cases of idiopathic hyperprolactinemia, spontaneous Prl release has been shown to be significantly elevated in obese women in direct proportion to the size of the visceral fat mass [22]. Because Prl is inhibited by activation of the dopamine $D_{2}$ receptor $\left(D_{2} R\right)$, increased Prl secretion may occur due to reduced $D_{2} R$ availability in the brain, which makes these individuals more likely to have elevated Prl secretion [6]. In a study considering hyperprolactinemia as a result of overweight, elevated Prl secretion in obese women was reported to be significantly reduced after loss of $50 \%$ of overweight [22]. In that study, the authors suggested that improvement of deficit $\mathrm{D}_{2} \mathrm{R}$-mediated neurotransmission and/or decreased circulating leptin/estrogen levels might be involved in this phenomenon. Weight reduction, with accompanying decrease in insulin levels, has been shown to lead to a normalization of Prl response in most, but not all, circumstances [10]. In a study with obese subjects, no significant association was found between baseline Prl levels and the degree of obesity in these patients [23]. One year after gastric bypass surgery, and consequent massive weight loss, no significant changes in the concentrations of the hormone were found, suggesting that Prl alone plays no role in the pathophysiology of obesity. Thus, although the magnitude of the effect of weight gain on Prl levels is still controversial, varying degrees of weight gain are commonly observed in patients with prolactinoma and, as reported in the present study, also in patients with other causes of hyperprolactinemia.

The association between Prl, weight gain and obesity suggests that Prl may play a role in the modulation of body weight and composition. However, it remains unclear whether weight gain is associated with hyperprolactinemia due to stimulation of lipogenesis or due to disruption of central nervous system (CNS) dopaminergic tone [24].

In this study, the prevalence of obesity was significantly high, regardless of the degree of obesity, in hyperprolactinemia of various causes. We detected a higher prevalence of obesity in patients with macroprolactinomas, compared to those with microprolactinomas and other causes of hyperprolactinemia. It is therefore important to monitor BMI in hyperprolactinemic patients in order to evaluate the influence of treatment of hyperprolactinemia on these variables and to introduce therapeutic intervention in obesity aiming to reduce associated comorbidities and maintain quality of life.

\section{Competing interests}

The authors declare that they have no competing interests.

\section{Authors' contributions}

JFSP, CGSL and MCO conceived and designed the research. FMF, MVRB and ALMS acquired the data. JFSP and CGSL analyzed and interpreted the data. FMF, MVRB and ALMS performed statistical analysis. MCO handled funding and supervision. JFSP, CGSL and MCO drafted the manuscript. JFSP, CGSL and MCO made critical revision of the manuscript for important intellectual Content. 
Pereira-Lima et al. Research Journal of Endocrinology and Metabolism 2013,

Publication history

Editor: Raul M Laque, University of Cordoba, Spain.

Received: 05-Apr-2013 Revised: 17-Jun-2013

Accepted: 30-Jul-2013 Published: 05-Aug-2013

\section{References}

1. Ben-Jonathan N, Mershon JL, Allen DL and Steinmetz RW. Extrapituitary prolactin: distribution, regulation, functions, and clinical aspects. Endocr Rev. 1996; 17:639-69. | Article | PubMed

2. Freeman ME, Kanyicska B, Lerant A and Nagy G. Prolactin: structure, function, and regulation of secretion. Physiol Rev. 2000; 80:1523-631. Article I PubMed

3. Ling C, Hellgren G, Gebre-Medhin M, Dillner K, Wennbo H, Carlsson $\mathrm{B}$ and Billig $\mathrm{H}$. Prolactin (PRL) receptor gene expression in mouse adipose tissue: increases during lactation and in PRL-transgenic mice. Endocrinology. 2000; 141:3564-72. | Article | PubMed

4. Ling $C$ and Billig $H$. PRL receptor-mediated effects in female mouse adipocytes: PRL induces suppressors of cytokine signaling expression and suppresses insulin-induced leptin production in adipocytes in vitro. Endocrinology. 2001; 142:4880-90. | Article | PubMed

5. Freemark M, Fleenor D, Driscoll P, Binart N and Kelly P. Body weight and fat deposition in prolactin receptor-deficient mice. Endocrinology. 2001; 142:532-7. | Article | PubMed

6. Kok P, Roelfsema F, Frolich M, Meinders AE and Pijl H. Prolactin release is enhanced in proportion to excess visceral fat in obese women. J Clin Endocrinol Metab. 2004; 89:4445-9. | Article | PubMed

7. Nilsson L, Binart N, Bohlooly YM, Bramnert M, Egecioglu E, Kindblom J, Kelly PA, Kopchick JJ, Ormandy CJ, Ling C and Billig H. Prolactin and growth hormone regulate adiponectin secretion and receptor expression in adipose tissue. Biochem Biophys Res Commun. 2005; 331:1120-6. | Article | PubMed

8. Ben-Jonathan N, Hugo ER, Brandebourg TD and LaPensee CR. Focus on prolactin as a metabolic hormone. Trends Endocrinol Metab. 2006; 17:110-6. | Article | PubMed

9. Weyer C, Funahashi T, Tanaka S, Hotta K, Matsuzawa Y, Pratley RE and Tataranni PA. Hypoadiponectinemia in obesity and type 2 diabetes: close association with insulin resistance and hyperinsulinemia. J Clin Endocrinol Metab. 2001; 86:1930-5. I Article I PubMed

10. Kopelman PG. Physiopathology of prolactin secretion in obesity. Int J Obes Relat Metab Disord. 2000; 24 Suppl 2:S104-8. | Article I PubMed

11. Creemers LB, Zelissen PM, van 't Verlaat JW and Koppeschaar HP. Prolactinoma and body weight: a retrospective study. Acta Endocrinol (Copenh). 1991; 125:392-6. I Article | PubMed

12. Greenman $\mathrm{Y}$, Tordjman $\mathrm{K}$ and Stern N. Increased body weight associated with prolactin secreting pituitary adenomas: weight loss with normalization of prolactin levels. Clin Endocrinol (Oxf). 1998; 48:547-53. | Article I PubMed

13. Doknic M, Pekic S, Zarkovic M, Medic-Stojanoska M, Dieguez C, Casanueva F and Popovic V. Dopaminergic tone and obesity: an insight from prolactinomas treated with bromocriptine. Eur J Endocrinol. 2002; 147:77-84. | Article | PubMed

14. dos Santos Silva CM, Barbosa FR, Lima GA, Warszawski L, Fontes R, Domingues RC and Gadelha MR. BMI and metabolic profile in patients with prolactinoma before and after treatment with dopamine agonists. Obesity (Silver Spring). 2011; 19:800-5. | Article | PubMed

15. Berinder K, Nystrom T, Hoybye C, Hall K and Hulting AL. Insulin sensitivity and lipid profile in prolactinoma patients before and after normalization of prolactin by dopamine agonist therapy. Pituitary. 2011; 14:199-207. | Article I PubMed

16. Delgrange $E$, Donckier J and Maiter D. Hyperprolactinaemia as a reversible cause of weight gain in male patients? Clin Endocrinol (Oxf). 1999; 50:271. | Article | PubMed

17. Soran $\mathrm{H}$, Wilding $\mathrm{J}$ and MacFarlane I. Body weight and prolactinoma: a retrospective study. Int J Obes Relat Metab Disord. 2004; 28:183. |
PubMed

18. Klibanski A. Clinical practice. Prolactinomas. N Engl J Med. 2010; 362:1219-26. | Article | PubMed

19. Bernichtein S, Touraine $P$ and Goffin V. New concepts in prolactin biology. J Endocrinol. 2010; 206:1-11. I Article I PubMed

20. Brazilian Ministry of Health. Brazilian Institute of Geography and Statistics (IBGE). Coordenação de Índices de Preços, Pesquisa de Orçamentos Familiares 2008/09 [Consumer Price Index, Household Budget Survey]. | Website

21. Melkersson K, Berinder K and Hulting AL. Effect of antipsychotic-induced hyperprolactinemia on anthropometric measures, insulin sensitivity and lipid profile in patients with schizophrenia or related psychoses. Neuro Endocrinol Lett. 2011; 32:428-36. I PubMed

22. Kok P, Roelfsema F, Langendonk JG, de Wit CC, Frolich M, Burggraaf J, Meinders $\mathrm{AE}$ and $\mathrm{Pijl} \mathrm{H}$. Increased circadian prolactin release is blunted after body weight loss in obese premenopausal women. Am J Physiol Endocrinol Metab. 2006; 290:E218-24. I Article | PubMed

23. Ernst $B$, Thurnheer $M$ and Schultes $B$. Basal serum prolactin levels in obesity--unrelated to parameters of the metabolic syndrome and unchanged after massive weight loss. Obes Surg. 2009; 19:1159-62. | Article | PubMed

24. Shibli-Rahhal A and Schlechte J. The effects of hyperprolactinemia on bone and fat. Pituitary. 2009; 12:96-104. | Article | PubMed

\section{Citation:}

Pereira-Lima J F, Leães C G, Freitas Neto F M, Barbosa M V, da Silva A L M and Oliveira M d C. Hyperprolactinemia and body weight: prevalence of obesity and overweight in patients with hyperprolactinemia. Res J Endocrinol Metab. 2013; 1:2. http://dx.doi.org/10.7243/2053-3640-1-2 